\title{
sciendo
}

\section{Motor Control: A Young Field with Many Facets (Introduction to the Special Issue)}

\author{
by \\ Grzegorz Juras ${ }^{1}$, Mark L. Latash ${ }^{2}$
}

\section{Past: A brief history of motor control}

Motor control has been defined as a field of natural science that searches for laws of nature that govern interactions between the central nervous system (CNS), the rest of the body, and the environment during biological movements. This topic has a long history, although it was addressed in earlier times using terms unusual for the contemporary scientific discourse. In particular, in ancient Greece, similar problems have been addressed as: "How does the soul control the body"? Aristoteles and Plato contributed significantly to the development of this field and, in particular, they compared the soul to the charioteer who uses a set of reins to produce actions of horses associated with individual body parts. The formulation of the main problem as that of interactions between the soul and the body persisted for close to two thousand years and, in fact, is alive at our times, although contemporary scientists prefer to use "mind" instead of "soul". Since both mind and soul are neither well-defined nor objectively observable, it is impossible to address such questions in terms of laws of nature.

A quiet revolution happened in Moscow in the 1960s. It was so quiet that most researchers outside the Moscow group failed to notice it for dozens of years and some continue to ignore it even now. This revolution was due to the efforts by three brilliant scientists, a physiologist Nikolai Bernstein, a mathematician Israel Gelfand, and a physicist Michael Tsetlin. Inspired by Bernstein's ideas, Gelfand and Tsetlin organized a seminar, which turned into an attractor for young, talented physiologists and physicists interested in problems of interaction between the CNS, the body, and the environment. This seminar led to the emergence of a number of Laboratories performing both experimental and theoretical studies in the field of the neural control of movements. Among those highly influential works, we would like to single out studies performed by Anatol Feldman in the mid-1960s, which, in our opinion, inaugurated the field of motor control as part of the natural science. In those studies, Feldman introduced the equilibrium-point (EP) hypothesis that viewed the neural control of a muscle as the process of setting time course of a neurophysiological parameter (threshold of the stretch reflex) defining the relation between muscle active force and length a law of nature common for all skeletal muscles across individuals and species.

In the 1970s, interest to problems within the field of motor control started to spread over the World, partly thanks to cracks in the Iron Curtain, resulting in better communication among researchers from the Soviet Union and other countries. We would like to mention the very important role of the series of motor control meetings in Bulgaria organized by Alexander Gidikov and Gancho Ganchev in the 1970s-1990s. It was possible, although not easy, to get

1 - Institute of Sport Sciences, The Jerzy Kukuczka Academy of Physical Education, Katowice, Poland.

2 - Department of Kinesiology. The Pennsylvania State University, University Park, PA, USA. 
permission to travel from the Soviet Union to Bulgaria, which was also open to visitors from the Western countries. During those times, two journals started to publish papers in the field of motor control on a regular basis, "Journal of Motor Behavior" led by Scott Kelso, Ron Marteniuk, Karl Newell, and Richard Schmidt and "Human Movement Science" shaped by John Whiting. In particular, John Whiting published a brief information letter on the First World Motor Control Day (9/9/93), which had been inaugurated in New Zealand.

Bernstein's centenary in 1996 signified another important milestone in the development of motor control into a full-fledged field of natural science. To honor Bernstein, a meeting was held in the Pennsylvania State University. This meeting was originally planned as a singular special event. Ultimately, however, this meeting became the first in the series "Progress in Motor Control", which are now biennial meetings of the International Society of Motor Control. Soon thereafter, a journal "Motor Control" has been established, followed by the International Society of Motor Control, and annual Motor Control Summer School.

At about the same time, young, energetic researchers from the Academy of Physical Education in Katowice (Poland) decided to initiate a series of motor control meetings in their country. The first couple of those meetings, in 2000 and 2004, were relatively modest in size and scope. Over the years, however, this series of meetings has turned into a major international event in basic and applied motor control attracting participants from all over the World. At about the same time, "Journal of Human Kinetics", also led by researchers from Poland, has developed from a purely applied behavioral journal into a much broader publication with substantial representation of motor control studies. Currently, "Journal of Human Kinetics" enjoys growing respect in the international scientific community, which is reflected in its impact factor.

We were eagerly expecting an even more successful Motor Control meeting in September of 2020, but the COVID-19 pandemic forced the organizers to cancel the meeting. This Special Issue, however, will play the role of a humble reassurance that there is a strong tradition and even stronger determination to keep up research in the field of motor control running.

\section{Present: Different facets of motor control}

The aforementioned groundbreaking studies of Feldman in the 1960s were followed by active development of the EP-hypothesis to multimuscle, multi-joint and whole-body movements. Now this approach is known as the theory of control with spatial referent coordinates. This is an actively developing field with applications to a variety of effector sets, tasks, and populations. It has transcended motor control and offered applications to related areas such as kinesthetic perception and cognition. We would also like to emphasize the highly promising and important applications of this theory to disordered movements and motor rehabilitation in neurological patients.

Another area of intensive studies, tightly linked to the idea of control with referent coordinates, in related to motor coordination. Problems of coordination emerge when a relatively large number of elements are involved in a motor task characterized by a relatively low number of task constraints thus allowing for numerous solutions. These problems are related to the famous problem of motor redundancy formulated and developed by Bernstein many years ago. A particular approach to studies of coordination among sets of effectors was developed in the 1970s-1980s based on ideas and tools from the area of dynamical systems introduced by Scott Kelso, Peter Kugler, and Michael Turvey, which emphasized the timevarying interaction between effectors and also with the environment leading to the formation of stable movement patterns. Later, this approach led to the introduction of the concept of uncontrolled manifold (UCM), readily compatible with the idea of motor abundance. The UCM concept is currently one of the most vigorously developed and explored in the field of motor control. In particular, it led to the emergence of a toolbox to analyze stability of functional movements produced by abundant sets of multiple elements. There have been promising examples of application of this method to analysis of patients with disordered coordination and impaired stability of movements. 
We would like to emphasize here the crucially important contribution of Dr. John Scholz, a brilliant scientist and true gentleman who passed away several years ago. John was the main driving force behind the early, active development of the UCM concept and its applications. He performed numerous seminal studies and trained many younger colleagues who are currently active in the field of motor control.

In its development, the field of motor control has been intertwined with other fields of movement studies. The emergence of new tools and major discoveries in related areas have played an important role in the formation of ideas in motor control. We cannot possibly mention here all the important contributions made over the past years in the fields of neurophysiology, biomechanics, and psychology. The field of motor control would not be where it is now without the works by Apostolos Georgopoulos, Sten Grillner, Jim Houk, John Rothwell, David Rosenbaum, Michael Turvey, and Vladimir Zatsiorsky. In addition, important insights have come from studies in applied fields such as motor learning and adaptation, top athletic performance, motor disorders, robotics and prosthetics. This Special Issue contains a mix of theoretical, experimental, and clinical papers representing many of the mentioned fields of movement studies.

\section{Future: Motor control in Poland and beyond}

Motor control is a very young field, wide open for exciting research and discoveries. As mentioned in the opening phrase, this field is based on laws of nature. As once said by one of the most famous physicists of the XX-th century, Erwin Schrödinger: "If you suspend laws of nature in the human body, you can explain anything." This sarcastic statement is equivalent to saying: "...you can explain nothing." That is why, with all due respect to such fields as mechanics and control theory, we would like to warn against overemphasizing their importance for motor control. Of course, biological movements do not violate laws of classical mechanics, but these laws, by themselves, are unable to predict what an animal would do in most natural situations. In other words, in contrast to movements of inanimate objects, laws of classical mechanics only constrain biological movements but do not prescribe them. Control theory was not developed to deal with issues of the neural control of movement. It has been highly powerful when applied to artificial, man-made objects, from ballistic missiles to robots. Applications of the control theory to biological movements have assumed that structures within the body perform computations relevant to interactions among different body parts and of those body parts with the environment. Frequently, such assumptions involve computing integrals of salient variables into future. These are very strong assumptions that border with "suspending laws of nature" as in the presented quotation from Schrödinger.

It is instructive that such internal computations are never assumed in fields of physiology that study other body functions such as cardiac physiology, processes of digestion, membrane physiology, muscle physiology, etc. No "internal model" of the liver is presumed to exist anywhere within the CNS. However, when the brain starts to play a role, it is very tempting to assume that there are magical processes in this enigmatic structure resulting in the computation of all the needed time function of hypothetical neural variables based on desired mechanics. An alternative, of course, is searching for interactions among neurons based on laws of nature that can form the basis for different stages in the process of generating voluntary movements, from selecting a target, to defining the timing of the action, and to ensuring its stability by coordinated involvement of numerous elements such as limbs, joints, digits, muscles, motor units, etc. Arguably, the most promising approach to this set of problems has been developed by the group of Gregor Schöner in the form of the dynamical field theory.

Recent developments of the field of motor control and related fields in Poland have been truly remarkable. In addition to the mentioned initiatives such as the series of Motor Control conferences and the development of the "Journal of Human Kinetics", Polish colleagues hosted the Motor Control Summer School and organized a number of workshops focused on motor control. We would like to emphasize the invaluable contributions of two persons to the development of movement studies in Poland who, sadly, passed away not that long ago.

Professor Joachim Raczek was the 
founder of the Department of Motor Behavior at the Academy of Physical Education. He opened the Academy for influences from the scientific world. He was a real Spiritus movement of huge developmental changes in Polish science, especially in Katowice. As a scientist, he focused on the endurance conditioning in children and youth, as well as in elite athletes. Professor Raczek dedicated the last two decades of his career to the area of human motor behavior. He introduced and developed the term "coordination abilities" to address the balanced process of growth, maturation, and physical activity. These so-called "coordination abilities", which have been recognized broadly in several European countries such as Germany, Czech Republic and Russia, still feature in many physical education and sport training handbooks. At the same time, Professor Raczek developed the concept of process background of coordination abilities. He and his co-workers were the first to introduce the UCM concept in Polish (in translation: niezależna podprzestrzeń współzależnych elementów). Professor Raczek spent a few years in German Universities, and some theories proposed by Kurt Meinel, Paul Baltes and other German scientists were close to his philosophy of science. A very special place in his mind was reserved for theories advanced by Nikolai Bernstein. Professor Joachim Raczek passed away in January 2019.

The motor control community as a whole suffered a major loss with passing away of our very close personal friend, brilliant scientist, Slobodan Jaric. Slobodan was at the peak of scientific productivity, full of new ideas, and starting new collaborations. He retired from his long-standing position in the University of Delaware and moved to his home in Belgrade to assume two positions, in the University of Belgrade and in the Academy of Physical Education in Katowice. He was in the process of developing new methods to test muscle mechanical capacities, which immediately attracted attention of the scientific community, and simultaneously was very active as an instructor and mentor. We miss badly his contribution to this Special Issue.

Although Poland has not yet reached the position of the scientific center of the Universe, the curiosity-driven research and determination of the faculty and students have led to Katowice being recognized as an important center of motor control studies. At the same time, several research groups working in Poznan, Wrocław and Warszawa are becoming major contributors to the field of movement studies. We hope that the tradition of motor control conferences in Poland. will go on beyond this Special Issue for years to come. 